\title{
Type 2 diabetes in adolescents: a severe phenotype posing major clinical challenges and public health burden
}

\section{Authors}

Russell Viner $\mathrm{PhD}^{1,2}$ (professor)

Billy White $\mathrm{PhD}^{1,2}$

Deborah Christie $\mathrm{PhD}^{2,3}$ (professor)

Affiliations

1: UCL Great Ormond Street Institute of Child Health. 30 Guilford St. London WC1N 1EH, UK

2: Child and Adolescent Diabetes Service, University College London Hospitals NHS Foundation Trust, 250 Euston Rd. London NW1 2PG, UK

3: UCL Institute of Epidemiology \& Healthcare. 1 - 19 Torrington Place, London WC1E 6BT, UK

Correspondence

Prof. Russell Viner

UCL Great Ormond St. Institute of Child Health 30 Guilford St.

London WC1N 1EH

r.viner@ucl.ac.uk 


\section{Summary}

Type 2 diabetes (T2D) in adolescence manifests as a severe progressive form of diabetes that frequently presents with complications, responds poorly to treatment and results in rapid progression of microvascular and macrovascular complications. Whilst overall still a rare disease, adolescent T2D now poses major challenges to paediatric and adult diabetes services across many countries. Therapeutic options are heavily curtailed by a lack of knowledge about the condition, with low numbers and poor trial recruitment impeding research. Together with lifestyle modification, metformin remains the first line therapy, although the majority rapidly progress to treatment failure and insulin therapy. Early bariatric surgery is controversial but has great potential to transform outcomes. Health systems must respond by both concentrating patients in specialist clinical services integrated with translational research programmes, but also joined-up with local health and social care services to improve engagement and take up of services. 


\section{Introduction}

Type 2 diabetes (T2D) in adolescence is rapidly becoming one of the paradigmatic disorders of the twenty-first century. Historically T2D was a disorder of ageing, never seen in children or young people (CYP) outside of rare genetic variants. T2D is now well described in adolescents across the globe, resulting from the epidemiological transition from infectious diseases to non-communicable diseases (NCDs) and injuries, driven by changes in nutrition, sanitation and health related to rapid economic development and social change over the last decades of the twentieth century. Adolescent T2D is prototypical of the emerging burden of NCDs related to obesity risk, largely affecting high income countries but rapidly evolving across middle and even low income countries.

The emergence of adolescent T2D presents new challenges for health systems. Adequately managing this new problem requires a paradigm shift for paediatric diabetes services and for the training of health professionals, hitherto almost entirely focused upon the much more common type 1 diabetes (T1D) and dominated by technological advances (pumps, sensors) that are less relevant to T2D.

In undertaking this seminar, we have focused on what is unique or particular to T2D in adolescence, using the World Health Organisation (WHO) definition of 10-19 years. Whilst young adults with T2D have also experienced a rapid rise in prevalence of T2D, and share many commonalitites with adolescents, we do not dwell on areas common to adult T2D.

We searched the Cochrane Library and PubMed on 11 January 2017. We used the PubMed search terms (("Diabetes Mellitus, Type 2"[Mesh]) AND (("Child"[Mesh] OR "Adolescent"[Mesh])) and similar terms in the Cochrane Library, restricting our search to the past 5 years. Review articles are cited to provide readers with further detail and references.

\section{Aetiology and phenotype}

Adolescent T2D is likely to be caused by a similar range of defective pathways proposed in adult T2D including $\beta$ cell failure, insulin resistance in liver and changes in incretin and pancreatic $\alpha$-cell function, kidney glucose filtration and lipolysis. ${ }^{1}$ There is evidence of exaggerated insulin insensitivity and rapid deterioration in $\beta$ cell function in adolescent T2D. ${ }^{2}{ }^{3}$ Adolescents have severe insulin insensitivity, but show a greater loss of glucose-stimulated insulin secretion, with a 2 to 4 fold faster decline in $\beta$ cell function than seen in adults. ${ }^{2}$ Estimates suggest that $\beta$ cell function may decline up to $20-35 \%$ per year on average, ${ }^{3}$ with little change in insulin insensitivity over the same period. This rapid progression of $\beta$ cell dysfunction likely explains the faster time to failure of metformin monotherapy in adolescents ${ }^{4}$ compared with adults. The frequent emergence of T2D during puberty likely reflects the transient $25-30 \%$ reducing in insulin sensitivity during puberty. ${ }^{5}$ The increased frequency of T2D during puberty likely reflects the transient $25-30 \%$ reduction in insulin sensitivity during puberty. ${ }^{5}$ Obesity is near universal in adolescents with $T 2 D^{6,7}$ and increases risk for T2D earlier in life. ${ }^{8}$

Our knowledge of risk factors has been dramatically expanded by recent large cohort studies from North America. The TODAY cohort ${ }^{9}$ was $65 \%$ female, ethnically highly diverse (largely from nonwhite groups: $41.1 \%$ Hispanic, $31.5 \%$ non Hispanic black) and predominantly from financially deprived backgrounds. Similar characteristics were observed in the Pediatric Diabetes Consortium (PDC) from 8 US academic centres, ${ }^{7,10}$ a population-based Canadian cohort ${ }^{6}$ and a national study across England and Wales ${ }^{11}$ (see Figure 1). 
Positive family histories of diabetes are nearly $90 \%$ within the first or second generation. ${ }^{9}$ Genetic studies of T2D have been confined to adults, however it is likely that similar genetic factors also operate in adolescents. ${ }^{12}$ Risk related to ethnicity is likely to represent genetic issues including metabolic differences as well as a range of complex cultural and social factors including deprivation. ${ }^{11}$

One-third of adolescents with T2D are born to mothers with $\mathrm{GDM}^{9}$ and are diagnosed at a younger age and with poorer B-cell function and higher $\mathrm{HbA}_{1 c} \cdot{ }^{13}$ It is likely that this reflects shared environments and genetic predisposition.

Observational epidemiology has identified a number of other potential risk factors:

- Those born at low birthweight and with rapid weight gain in childhood appear most at risk for T2D in young adult and adult life. ${ }^{14,15}$

- The very strong association of adolescent T2D with low socio-demographic status is likely mediated partly through the association of deprivation with obesity, but also through stress and its concomitant mental health disorders such as depression, which increase T2D risk. ${ }^{16,17}$

- A recent systematic review concluded that antipsychotic-exposed youth had a cumulative T2D risk of 5.7 per 1000 patients, many-fold higher than the general population and in psychiatric controls. ${ }^{18}$ Risk relates to rapid weight gain but may also relate to effects on insulin sensitivity and the hypothalamus. ${ }^{19}$ Atypical antipsychotic prescribing to children and adolescents increased 60-fold in the UK from the mid1990s to reach 0.61 users per 1000 patient-years in $2005 .{ }^{20}$

\section{Epidemiology}

The extent of the burden of T2D in adolescence remains unclear. The only recent national prevalence rates are available from the UK $\left(2.9 / 100,000\right.$ for under 16 year olds) ${ }^{11}$ and from Denmark, where a register study identified only 7 adolescents nationally with T2D. ${ }^{21}$ In Europe, T2D meets criteria for being a rare disease (prevalence $<5 / 10,000$ ).

Variation in incidence and prevalence across countries is striking. A systematic review reported an incidence range of $0-330$ per 100,000 person-years and prevalence ranging from $0-5,300$ per 100,000 adolescents, although many studies were from limited samples and single ethnic groups. ${ }^{22}$ The highest prevalence estimates were in Pima Indian and First Nation groups in North America. ${ }^{22}$

The lowest incidence rates were observed in European countries, over ten-fold lower than in the USA. The most recent national paediatric diabetes audit in England and Wales identified 533 adolescents with T2D, making up approximately $2 \%$ of all child and adolescent diabetes. ${ }^{23}$

Longitudinal sub-national US data suggest that prevalence increased significantly by $7 \%$ annually during the twenty-first century, reaching 12.5 cases per 100,000 in 2011-12, with annual increases higher amongst black, Asians or Pacific Islanders, and Native Americans. ${ }^{24} \mathrm{~T} 2 \mathrm{D}$ is reported to account for $10-50 \%$ of new onset adolescent diabetes cases in the USA, ${ }^{25,26}$ and over half amongst minority ethnic groups. ${ }^{27}$

Few data are available outside the USA and Europe. Incidence of adolescent T2D doubled in two Korean regions between 2001 and 2010. ${ }^{28}$ Isolated reports from Asia suggest a rising prevalence of early onset T2D, ${ }^{29}$ with around $2-3 \%$ of T2D with onset under 20 years in south and east $\mathrm{Asia}^{30}$ and the Arab world. ${ }^{31}$ 


\section{Diagnosis and differentiation from other forms of diabetes}

The standard diagnostic criteria for diabetes are relevant for all forms of diabetes in adolescence, and are now based largely upon $\mathrm{HbA}_{1 \mathrm{c}}$ values following a report from an International Expert Committee. ${ }^{32}$ These recommendations are based solely on evidence from adults which demonstrates a good correlation between $\mathrm{HbA1c}$ and diabetes-related complications. Screening using $\mathrm{HbA1c}$, random- and fasting-glucose levels detect different populations $\mathrm{s}^{33}{ }^{34}$ leading to calls for a "mixed testing" approach using a combination of these measures in adolescents (an approach which the Expert Committee argued against). The International Society of Paediatric \& Adolescent Diabetes (ISPAD) and ADA guidelines recommend screening using either fasting-, random-, 2-hour post-challenge glucose or $\mathrm{HbA}_{1 \mathrm{c}}$ : ${ }^{35} 36$

- Random-, fasting-, and 2 hour post-challenge glucose thresholds remain unchanged. Diabetes should not be diagnosed solely on a random plasma glucose concentration $>11.1$ $\mathrm{mmol} / \mathrm{l}$ (>200 mg/dl) without classical symptoms of diabetes.

- The diagnosis of diabetes is made if the $\mathrm{HbA}_{1 \mathrm{c}}$ level is $\geq 6.5 \%$ ( $\left.48 \mathrm{mmol} / \mathrm{mol}\right)$. ADA recommend a raised $\mathrm{HbA} 1 \mathrm{c}$ should be confirmed with a second $\mathrm{HbA}_{1 \mathrm{c}}$ test. ${ }^{36}$ Further, they consider an $\mathrm{HbA}_{1 \mathrm{c}}$ between $5.7 \%$ and $6.5 \%$ to represent a prediabetic state.

The presumptive diagnosis related to persistent hyperglycaemia and its symptoms (e.g. thirst, polyuria, weight-loss and fatigue) in adolescents should be T1D, unless there are strong indications for other forms of diabetes. ${ }^{37}$ However, T2D should be considered in all incident cases, particularly when the clinical picture is unusual or there are features consistent with T2D. The classic features of T2D in adolescence are obesity, evidence of insulin resistance (e.g. acanthosis nigricans, seen in $86 \%$ in the TODAY cohort ${ }^{9}$ ), lack of auto-antibodies and a strong family history of T2D with ethnic minority groups most at risk (see Figure 1). ${ }^{38}$

Severe acute presentations of T2D are uncommon. Only $67 \%$ present with symptoms of diabetes with one third identified through screening of at-risk adolescents. ${ }^{10}$ Acute symptoms at presentation are rarely those of diabetic ketoacidosis (DKA), found in only $6^{39}$ to $11 \%{ }^{10}$ of incident cases. Reports that DKA is an increasing presentation amongst adolescent T2D appear misplaced, as the prevalence appears to have halved over the past $10-15$ years. ${ }^{39}$

Presentation with diabetic hyperglycaemic hyperosmolar syndrome (HHS; see Figure 2) is rare (e.g. $2 \%),{ }^{10}$ with most adolescent cases due to T1D. ${ }^{40}$

Differentiation of T1D and T2D can be difficult in overweight young people who present with hyperglycaemia without ketosis. Modern adolescents with autoimmune T1DM may also be overweight or obese at diagnosis, and the commonality of adult T2D means that a positive family history is often seen in T1D. Pre-pubertal adolescents are unlikely to have T2D even if obese. ${ }^{35}$ The presence of diabetes complications at presentation is relatively common in T2D although very rare in T1D, and likely reflects long periods of asymptomatic hyperglycaemia before diagnosis.

Differentiation of T2D from maturity-onset diabetes of the young (MODY) can be difficult in obese antibody-negative adolescents, ${ }^{41}$ and genetic testing should be performed if the phenotype is unclear (see Figure 3). The presence of diabetes-specific autoantibodies at diagnosis is strongly suggestive of T1D, however $10 \%$ or more of those diagnosed with T2D can have autoantibodies, leading to uncertainty about the diagnosis. ${ }^{9} \mathrm{C}$-peptide is of little value at presentation although may be of value some months later if there is difficulty in finalising the diagnosis in those on insulin. ${ }^{42}$ 
Our understanding of the disease process in adolescents and the few data available suggest that the nature of complications are similar to those seen in adult T2D and in T1D. However the overall risk of complications is much higher than in T1D adolescents, with complications appearing earlier in the disease process. ${ }^{6}$ Longitudinal Canadian data suggest that macrovascular peripheral vascular complications began to occur 15 years after diagnosis in adolescent T2D. ${ }^{6}$ Adult cardiovascular risk tools are not informative for risk in adolescents with T2D.

Renal disease is the commonest and earliest complication of adolescent T2D, with a higher risk of progression than in childhood T1D or adult T2D. ${ }^{43}$ The prevalence of microalbuminuria (MA) has recently been reported to be $13 \%{ }^{9}$ to $27.1 \%^{6}$ at diagnosis and $16 \%{ }^{44}$ to $38.6 \%^{6}$ after $4-5$ years, with progression associated with $\mathrm{HbA}_{1 \mathrm{c}}{ }^{44}$ Concerningly, data from the TODAY trial suggested that that MA progressed over time regardless of treatment arm. ${ }^{44}$ Hypertension has been reported in $10^{6}$ to $12 \%^{44}$ at diagnosis, increasing to $34 \%^{44}, 36 \%^{45}$ to $45.8 \%^{6}$ after $4-5$ years, with those with higher BMI at greater risk. ${ }^{44}$

Little is known about the natural history of retinopathy in adolescent T2D, although there is some suggestion that progression is more rapid than in T1D. The largest and most representative cohort (TODAY) reported the prevalence of retinopathy was $13.7 \%$ after a mean duration of 5 years, ${ }^{46}$ although the much smaller SEARCH cohort reported $42 \%$ with retinopathy after an average of 7 years duration. ${ }^{47}$ Where identified, retinopathy was of low grade. Longer duration of diabetes and higher $\mathrm{HbA1c}$ predicted higher risk of retinopathy.$^{46}$ One report identified a $6 \%$ prevalence of cataracts. ${ }^{48}$

Dyslipidaemia, is the most prevalent and non-treatment responsive comorbidity of adolescent T2D. In the TODAY cohort at diagnosis using standard adult definitions of dyslipidaemia, $80.5 \%$ had low HDL-cholesterol, $10.2 \%$ had high triglycerides and $4.5 \%$ had significantly high LDL-cholesterol. ${ }^{9}$ Using definitions minimally altered for adolescents in the SEARCH cohort, $65.6 \%$ had high triglycerides and $61.5 \%$ had low HDL-cholesterol after 2 years of T2D..$^{49}$ TODAY reported that LDL rose across the cohort in line with $\mathrm{HbA1c}$ regardless of treatment, with the proportion having elevated LDL rising to $10.7 \%$ after 3 years. ${ }^{44}$

Data on neuropathy in adolescent T2D are extremely limited. A prevalence of 7.6\% after 6-7 years of diabetes ${ }^{6}$ was reported in a state-wide Canadian cohort.

Adolescents with T2D have extremely poor pregnancy outcomes, with high rates of fetal loss (22\%), preterm birth (15\%) and major congenital anomalies $(21 \%)$ in some cohorts. ${ }^{50}$ It remains unclear how much of these poor outcomes relate to the morbid obesity and deprivation associated with T2D.

The prevalence of significant depressive symptoms in adolescents with T2D is $15^{44}$ to $22 \%,{ }^{51}$ nearly twice as high as in T1D adolescents. ${ }^{51}$ Whilst females are most at risk, there is little evidence that $\mathrm{BMI}$ or deprivation increase risk. ${ }^{44}$ Depressed mood is associated with poorer glycaemic control, a greater number of visits to the emergency department and poor adherence to treatment recommendations..$^{52}$ Exposure to major stressful life events is also associated with impaired psychosocial functioning and lower adherence to oral medication regimens in adolescents with recent onset T2D. ${ }^{53}$

Neuropsychiatric comorbidity is also common in adolescent T2D, with one large cohort reporting the combined prevalence of depression, ADHD, neurodevelopmental disorders, schizophrenia and bipolar disorder in $19.4 \%$ of a sample of 237 adolescents with T2D, with $63 \%$ of those diagnosed on 
psychotropic medications. ${ }^{54}$ Clinically significant levels of binge eating have been reported in $6.2 \%$ of adolescents with T2D, with risk of binge eating associated with higher BMI. ${ }^{44}$

QOL appears lower in adolescents with T2D than those with T1D, ${ }^{55}$ with higher $\mathrm{BMI}$ and a greater number of comorbidities linked with lower QOL. ${ }^{56}$ However it remains unclear to what degree reduced QOL relates to T2D or to obesity, deprivation or depression, which are themselves associated with reduced QOL.

Adolescents with T2D show a wide range of emotional adjustment and levels of motivation for selfcare. Young people describe the need for support for diabetes specific behaviours and difficulty balancing competing interests. A consistent theme is a fear of disclosing their diagnosis to others. Parents report that the need for perceived normalcy is also factor in adjustment to T2D, and that adolescents with T2D have less problem-solving and coping skills; making sensible food choices is a common challenge. ${ }^{57}$ Witnessing negative health consequences in family members or friends with T2D can be an important source of motivation, ${ }^{58,59}$ but also a source of hopelessness.

\section{Management}

Core management should include intensive lifestyle modification comprising promotion of weight control through increased lifestyle activity, reduced sedentary behaviour and a healthy balanced diet with elimination if possible of simple sugars and reduced carbohydrate and total and saturated fat intake and increased fibre intake. ${ }^{35}$ Regular physical activity (PA) improves cardiorespiratory fitness, body composition, bone health, insulin sensitivity, and psychosocial well-being. However most adolescents with T2D do not meet recommended PA thresholds of 60 minutes of moderate exercise daily. ${ }^{60}$

Observational data shows that some can achieve and sustain glycaemic control through lifestyle change alone; ${ }^{61,62}$ however lifestyle change has not been compared to drug therapy alone in randomised trials. A further assumption has been that lifestyle change combined with drug therapy improves glycaemic control; however the lifestyle programme used in TODAY did not enable improved glycaemic control. ${ }^{4}$

Home management should include regular self-monitoring of capillary blood glucose (BG) in those on oral hypoglycaemic medications or insulin, although the necessary frequency when on oral medications is unclear. ${ }^{60}$

Care should be provided by a multi-disciplinary team, with psychologists, dietitians and potentially physical therapists as core members of the team alongside specialist doctors and nurses.

ISPAD suggests screening for all potential complications at diagnosis, including for obstructive sleep apnea (OSA), non-alcoholic related fatty liver disease (NAFLD) and depression as well as for pregnancy. ${ }^{35}$ Thereafter ISPAD suggests monitoring of HbA1c and blood pressure at each visit and annual monitoring of retinopathy, microalbuminuria, dyslipidaemia and liver function, with screening for OSA and depression less frequently. Smoking and other substance use should be part of annual screening. ${ }^{35}$

Treatment of T2D is challenging for three main reasons. First, T2D is likely to be an umbrella of diseases, with different genetic, environmental and metabolic triggers that are likely not to respond equally to treatments. ${ }^{2}{ }^{63}$ Furthermore, better understanding of drug action has shown individual pharmaco-genetic responses to drugs, including differing side-effect profiles for metformin ${ }^{64}$ and pharmacodynamics of sulphonylureas. ${ }^{65}$ 
Second, there are few evidence-based treatments available due to lack of studies in this age group. The TODAY study is the only RCT to date examining different treatment options in adolescents. ${ }^{66} \mathrm{It}$ compared metformin alone, metformin plus intensive lifestyle treatment and metformin plus rosiglitazone with baseline criteria including $\mathrm{HbA1c}<8 \%$ with metformin monotherapy. Metformin monotherapy resulted in treatment failure (defined as $\mathrm{HbA1C}>8 \%$ ) in half the group $(51.7 \%$ of cohort) with those allocated to additionally receive the TDZ rosiglitazone having reduced likelihood of treatment failure (38.6\%) but not those with an additional weight-loss intensive lifestyle programme $(46.6 \%) .{ }^{66}$

Metformin and insulin are currently the only drugs licenced for use in adolescent T2D. Guidelines from the American Diabetic Association (ADA), ISPAD and NICE each recommend metformin as first line treatment once initial metabolic derangement has resolved. ${ }^{36} 4235$ The TODAY run-in period regimen of metformin monotherapy, together with expert education and support showed that glycaemic control can be improved with this triad. At the same time, $24 \%$ of this run-in group were not eligible for the main study due to inadequate adherence (10.9\%), inability to achieve glycaemic control (6.3\%), need for polypharmacy (2.5\%) and metformin intolerance (2.0\%). ${ }^{67}$ Further treatments beyond metformin are needed.

ADA and ISPAD but not NICE recommend insulin as the only second line treatment, with first line use to reverse the initial metabolic derangement in those who present with ketosis. US guidelines suggest insulin initiation when $\mathrm{HbA} 1 \mathrm{c}$ is $\geq 9 \% .{ }^{35}$ This is despite insulin's cost, associated weight gain, injectable formulation ${ }^{68}$, risk of hypoglycaemia and lack of evidence in this age group. In comparison, many drugs are licenced for use in adults in the USA and Europe, including biguanides (metformin), sulfonlyureas (SU), thiazolidinediones (TDZ), GLP-1 receptor agonists, DPP-4 inhibitors, SGLT2 inhibitors, and insulins, ${ }^{36}$ although a number of these have failed to be licensed for adolescents. ${ }^{69}$

Recent data from the PDC cohort suggests over $90 \%$ of adolescent T2D were treated with insulin or metformin, with approximately $30 \%$ on each of metformin or insulin alone and one-third on both, with only $7 \%$ on diet/lifestyle alone. ${ }^{10}$ Yet the proportions on insulin rise with each duration year, reaching $60 \%$ in those with T2D for $\geq 4$ years. $^{70}$

Bariatric surgery is the only treatment currently available that results in significant weight loss and improved long term glycaemic control as well as improvements in blood pressure, lipids and sleep apnoea. ${ }^{71}{ }^{72}$ Bariatric surgery is now an accepted treatment for T2D in adults, although there is insufficient evidence to recommend it as a mainline treatment for adolescent $\mathrm{T} 2 \mathrm{D} . .^{73}$ In the largest US adolescent cohort, 19 of 20 with T2D were in remission 3 years after surgery. ${ }^{74}$ Of the three procedures widely used, the Roux-en-Y gastric bypass (GB) appears the most effective and most complex procedure. ${ }^{71}$ Its complexity is likely to drive its superior efficacy in that it influences more pathways that have been suggested (but not definitely proven) to promote weight loss including gut microbiota changes, bile acid and incretin metabolism and vagal nerve changes. ${ }^{75}$ The ADA, ISPAD and NICE are cautious in recommending bariatric surgery in adolescence given limited evidence and hypothesised risks including reduced bone mineral density. ${ }^{71,76} 77$ However, the magnitude of weight loss is incomparable with other interventions with good safety data ${ }^{74}$ making bariatric surgery a feasible option that should be encouraged in those with severe obesity and poorly controlled diabetes. Limited qualitative evidence suggests adolescents support surgery as a therapeutic option. ${ }^{68}$

Many patients, families and clinicians are reluctant to initiate insulin or undergo surgery. ${ }^{68}$ Clearly alternative treatment options are needed, and to this effect, ISPAD guidelines outline off-licence treatment options including DDP-IV antagonist and weight-beneficial GLP-1 agonists that both act on 
the incretin pathway to increase satiety and improve glycaemic control. GLP-1 agonists have similar tolerability, safety, ${ }^{78}$ and pharmacokinetics ${ }^{79}$ as in adults, although their place in treatment pathways for adolescents is yet to be established.

Blood pressure and lipid control are likely to be important to promote long term cardiovascular health. In the absence of robust evidence in this age group or expert consensus, clinicians should seek specialist guidance on treatment when age-appropriate thresholds for blood pressure or lipids are exceeded. Practice studies show lower concordance with BP and lipid than glucose recommendations, with lack of clinician familiarity a key barrier. ${ }^{80}$ This supports managing adolescent T2D in more specialist centres.

Lastly, T2D is associated with high familial disease burden (especially type 2 diabetes), socioeconomic deprivation and multiple co-morbidities including obesity. ${ }^{9} \mathrm{~A}$ small clinical study found that clinic attendance by adolescents with T2D was notably lower than desired by professionals and very poor attenders had the highest $\mathrm{HbA1c} .^{81}$ Lifestyle change is likely to be an important component of diabetes treatment however these conflicting needs likely make change difficult. The limited efficacy of lifestyle interventions in obesity likely reflects the complex interactions that influence behaviour, and broader interventions are likely to be needed including those at public health level.

\section{Prevention strategies}

Adequate management of adolescent T2D requires us to focus upstream from the individual young person to families and social systems and to the prevention of obesity. A discussion of preventive approaches to child and adolescent obesity is beyond the scope of this review, although there is emerging evidence that systemic approaches can halt or even reverse the rise of childhood obesity.

There is very limited evidence on limiting progression of prediabetes or T2D. A recent systematic review identified a range of community, school or clinical interventions which improved various risk factors (e.g. dietary or physical activity measures) and indices of cardiometabolic health and may contribute to prevention of T2D, ${ }^{82}$ although currently no studies have shown efficacy in prevention of incidence of T2D.

There is currently no evidence to support screening of obese adolescents for T2D. ${ }^{21}$ The vast majority of obese adolescents do not have T2D ${ }^{83}$ and do not develop it within adolescence

\section{Conclusion}

Adolescent T2D manifests with a severe and progressive phenotype, poor response to current treatments and major challenges in adherence and management.

Increases in prevalence driven by the obesity epidemic are likely to pose major challenges for future burden of disease. Adolescent T2D in adolescence is theoretically preventable in the current state of knowledge, unlike T1D. Yet targeted prevention efforts have yielded little benefit, and success will likely depend on broader systemic efforts to reduce obesity.

A major barrier to effective management is the lack of approved pharmacological agents and the lack of psychosocial or educational interventions of proven effectiveness. In common with other rare diseases this in part relates to poor visibility of the problem and the lack of patient numbers even in large centres. Added to this is also low participation in research trials. ${ }^{84}$ Even large national cohorts including the T2D in Childhood: UK National Cohort Study (JUMP study) in the UK, ${ }^{85}$ suffer from similar recruitment issues. 
We believe that adolescent T2D needs to be reframed as a severe progressive disorder, and adolescents with T2D recognised as a high risk group within both paediatric diabetes services and adult diabetes services, requiring innovative psychological and pharmacological approaches. Conceptualising T2D as a 'rare disease' may have benefits, highlighting its severity and the lack of service focus and available treatments for the condition. Rare diseases approaches therefore logical, concentrating patients in evidence-based specialist paediatric diabetes services that are integrated with world-class research with a focus on rapid translation of research findings into care and which are linked with adult diabetes services. Such services must actively work to share care with local services and primary care, potentially using novel telehealth technologies, although these are yet to be well-evidenced, to ensure integration of the health and social care systems around these vulnerable patients. Key research priorities include evaluation of safety, acceptability and effectiveness of newer pharmacological agents and combinations of agents, development of strategies for comorbidity management and cardiovascular risk minimization, interventions to promote adherence with regimens and facilitation of lifestyle change to enable weight loss and optimal methods of service delivery for this vulnerable group. 


\section{Competing interest statement}

Russell Viner has no conflict of interests to declare. In the past 10 years he has accepted no speaker fees or honoraria from phamarceutical or device companies. He has attended meals funded by Medtronic Ltd related to clinical training in insulin pump use.

Deborah Christie declares no relationships that pose a conflict of interest for this work. She has accepted speaker fees from Lily, other from Pfizer, Medtronic and Novo Nordisk for presentations relating to psychological issues in diabetes. As she is a psychologist, none of these relate to use of pharmaceutical agents or devices.

Billy White declares no relationships that pose a conflict of interest for this work. He has received speakers fees from Lily and Omniamed for presentations on transition in diabetes, none of which included use of pharmaceutical agents. 


\section{References}

1. DeFronzo RA. From the Triumvirate to the Ominous Octet: A New Paradigm for the Treatment of Type 2 Diabetes Mellitus. Diabetes 2009; 58(4): 773-95.

2. Hannon TS, Arslanian SA. The changing face of diabetes in youth: lessons learned from studies of type 2 diabetes. Annals of the New York Academy of Sciences 2015; 1353(1): 113-37.

3. Today Study Group. Effects of metformin, metformin plus rosiglitazone, and metformin plus lifestyle on insulin sensitivity and beta-cell function in TODAY. Diabetes Care 2013; 36(6): 1749-57.

4. Group TS, Zeitler P, Hirst K, et al. A clinical trial to maintain glycemic control in youth with type 2 diabetes. N Engl J Med 2012; 366(24): 2247-56.

5. Hannon TS, Janosky J, Arslanian SA. Longitudinal study of physiologic insulin resistance and metabolic changes of puberty. Pediatr Res 2006; 60(6): 759-63.

6. Dart AB, Martens PJ, Rigatto C, Brownell MD, Dean HJ, Sellers EA. Earlier onset of complications in youth with type 2 diabetes. Diabetes Care 2014; 37(2): 436-43.

7. Silverstein J, Cheng P, Ruedy KJ, et al. Depressive Symptoms in Youth With Type 1 or Type 2 Diabetes: Results of the Pediatric Diabetes Consortium Screening Assessment of Depression in Diabetes Study. Diabetes Care 2015; 38(12): 2341-3.

8. Wei GS, Coady SA, Reis JP, et al. Duration and Degree of Weight Gain and Incident Diabetes in Younger Versus Middle-Aged Black and White Adults: ARIC, CARDIA, and the Framingham Heart Study. Diabetes Care 2015; 38(11): 2042-9.

9. Copeland KC, Zeitler P, Geffner M, et al. Characteristics of adolescents and youth with recent-onset type 2 diabetes: the TODAY cohort at baseline. J Clin Endocrinol Metab 2011; 96(1): 159-67.

10. Klingensmith GJ, Connor CG, Ruedy KJ, et al. Presentation of youth with type 2 diabetes in the Pediatric Diabetes Consortium. Pediatr Diabetes 2016; 17(4): 266-73.

11. Khanolkar AR, Amin R, Taylor-Robinson D, Viner R, Warner J, Stephenson T. Ethnic Minorities Are at Greater Risk for Childhood-Onset Type 2 Diabetes and Poorer Glycemic Control in England and Wales. J Adolesc Health 2016; 59(3): 354-61.

12. Morgan AR. Determining genetic risk factors for pediatric type 2 diabetes. Current diabetes reports 2012; 12(1): 88-92.

13. Chernausek SD, Arslanian S, Caprio S, et al. Relationship Between Parental Diabetes and Presentation of Metabolic and Glycemic Function in Youth With Type 2 Diabetes:

Baseline Findings From the TODAY Trial. Diabetes Care 2016; 39(1): 110-7.

14. Eriksson JG, Kajantie E, Lampl M, Osmond C. Trajectories of body mass index amongst children who develop type 2 diabetes as adults. J Intern Med 2015; 278(2): 219-26. 15. Bhargava SK, Sachdev HS, Fall CH, et al. Relation of serial changes in childhood bodymass index to impaired glucose tolerance in young adulthood. N Engl J Med 2004; 350(9): 865-75.

16. Hannon TS, Rofey DL, Lee S, Arslanian SA. Depressive symptoms and metabolic markers of risk for type 2 diabetes in obese adolescents. Pediatr Diabetes 2013; 14(7): 497503.

17. Suglia SF, Demmer RT, Wahi R, Keyes KM, Koenen KC. Depressive Symptoms During Adolescence and Young Adulthood and the Development of Type 2 Diabetes Mellitus. Am J Epidemiol 2016; 183(4): 269-76. 
18. Galling B, Roldan A, Nielsen RE, et al. Type 2 Diabetes Mellitus in Youth Exposed to Antipsychotics: A Systematic Review and Meta-analysis. JAMA Psychiatry 2016; 73(3): 24759.

19. Pramyothin P, Khaodhiar L. Type 2 diabetes in children and adolescents on atypical antipsychotics. Current diabetes reports 2015; 15(8): 53.

20. Rani F, Murray ML, Byrne PJ, Wong IC. Epidemiologic features of antipsychotic prescribing to children and adolescents in primary care in the United Kingdom. Pediatrics 2008; 121(5): 1002-9.

21. Oester IM, Kloppenborg JT, Olsen BS, Johannesen J. Type 2 diabetes mellitus in Danish children and adolescents in 2014. Pediatr Diabetes 2016; 17(5): 368-73.

22. Fazeli Farsani S, van der Aa MP, van der Vorst MM, Knibbe CA, de Boer A. Global trends in the incidence and prevalence of type 2 diabetes in children and adolescents: a systematic review and evaluation of methodological approaches. Diabetologia 2013; 56(7): 1471-88.

23. National Paediatric Diabetes Audit 2014-15. London: Royal College of Paediatrics \& Child Health, 2016.

24. Mayer-Davis EJ, Lawrence JM, Dabelea D, et al. Incidence Trends of Type 1 and Type 2 Diabetes among Youths, 2002-2012. N Engl J Med 2017; 376(15): 1419-29.

25. Bobo N, Evert A, Gallivan J, et al. An update on type 2 diabetes in youth from the National Diabetes Education Program. Pediatrics 2004; 114(1): 259-63.

26. Petitti DB, Klingensmith GJ, Bell RA, et al. Glycemic control in youth with diabetes: the SEARCH for diabetes in Youth Study. J Pediatr 2009; 155(5): 668-72 e1-3.

27. Group SfDiYS, Liese AD, D'Agostino RB, Jr., et al. The burden of diabetes mellitus among US youth: prevalence estimates from the SEARCH for Diabetes in Youth Study. Pediatrics 2006; 118(4): 1510-8.

28. Lee JH, Kim YM, Kwak MJ, et al. Incidence trends and associated factors of diabetes mellitus in Korean children and adolescents: a retrospective cohort study in Busan and Gyeongnam. Ann Pediatr Endocrinol Metab 2015; 20(4): 206-12.

29. Praveen PA, Kumar SR, Tandon N. Type 2 diabetes in youth in South Asia. Current diabetes reports 2015; 15(2): 571.

30. Yeung RO, Zhang $\mathrm{Y}$, Luk A, et al. Metabolic profiles and treatment gaps in youngonset type 2 diabetes in Asia (the JADE programme): a cross-sectional study of a prospective cohort. Lancet Diabetes Endocrinol 2014; 2(12): 935-43.

31. Alsafar H, Jama-Alol KA, Hassoun AAK, Tay GK. The prevalence of Type 2 Diabetes Mellitus in the United Arab Emirates: justification for the establishment of the Emirates Family Registry. Int J Diabetes Dev Ctries 2012; 32(1): 25-32.

32. International Expert Committee. International Expert Committee report on the role of the A1C assay in the diagnosis of diabetes. Diabetes Care 2009; 32(7): 1327-34.

33. Lee JM, Gebremariam A, Wu EL, LaRose J, Gurney JG. Evaluation of nonfasting tests to screen for childhood and adolescent dysglycemia. Diabetes Care 2011; 34(12): 2597-602.

34. Nowicka P, Santoro N, Liu H, et al. Utility of hemoglobin A1c for diagnosing prediabetes and diabetes in obese children and adolescents. Diabetes care 2011; 34(6): 1306-11.

35. Zeitler P, Fu J, Tandon N, et al. ISPAD Clinical Practice Consensus Guidelines 2014. Type 2 diabetes in the child and adolescent. Pediatr Diabetes 2014; 15 Suppl 20: 26-46.

36. American Diabetes Association. Standards of Care. Diabetes Care 2017; 40(Supplement 1): S1-S135. 
37. Beckles ZL, Edge JA, Mugglestone MA, Murphy MS, Wales JK, Guideline Development G. Diagnosis and management of diabetes in children and young people: summary of updated NICE guidance. BMJ 2016; 352: i139.

38. Copeland KC, Zeitler P, Geffner M, et al. Characteristics of Adolescents and Youth with Recent-Onset Type 2 Diabetes: The TODAY Cohort at Baseline. The Journal of Clinical Endocrinology \& Metabolism 2011; 96(1): 159-67.

39. Dabelea D, Rewers A, Stafford JM, et al. Trends in the prevalence of ketoacidosis at diabetes diagnosis: the SEARCH for diabetes in youth study. Pediatrics 2014; 133(4): e93845.

40. Bagdure D, Rewers A, Campagna E, Sills MR. Epidemiology of hyperglycemic hyperosmolar syndrome in children hospitalized in USA. Pediatr Diabetes 2013; 14(1): 1824.

41. Pihoker C, Gilliam LK, Ellard S, et al. Prevalence, characteristics and clinical diagnosis of maturity onset diabetes of the young due to mutations in HNF1A, HNF4A, and glucokinase: results from the SEARCH for Diabetes in Youth. $J$ Clin Endocrinol Metab 2013; 98(10): 4055-62.

42. National Institute for Health and Care Excellence (NICE). Diabetes (type 1 and type 2) in children and young people: diagnosis and management. Nice guideline NG18: NICE, UK, 2016.

43. Bjornstad P, Cherney DZ, Maahs DM, Nadeau KJ. Diabetic Kidney Disease in Adolescents With Type 2 Diabetes: New Insights and Potential Therapies. Current diabetes reports 2016; 16(2): 11.

44. Tryggestad JB, Willi SM. Complications and comorbidities of T2DM in adolescents: findings from the TODAY clinical trial. J Diabetes Complications 2015; 29(2): 307-12.

45. Eppens MC, Craig ME, Cusumano J, et al. Prevalence of diabetes complications in adolescents with type 2 compared with type 1 diabetes. Diabetes Care 2006; 29(6): 1300-6. 46. Today Study Group. Retinopathy in youth with type 2 diabetes participating in the TODAY clinical trial. Diabetes Care 2013; 36(6): 1772-4.

47. Mayer-Davis EJ, Davis C, Saadine J, et al. Diabetic retinopathy in the SEARCH for Diabetes in Youth Cohort: a pilot study. Diabet Med 2012; 29(9): 1148-52.

48. Geloneck MM, Forbes BJ, Shaffer J, Ying GS, Binenbaum G. Ocular Complications in Children with Diabetes Mellitus. Ophthalmology 2015; 122(12): 2457-64.

49. Mayer-Davis EJ, Ma B, Lawson A, et al. Cardiovascular disease risk factors in youth with type 1 and type 2 diabetes: implications of a factor analysis of clustering. Metab Syndr Relat Disord 2009; 7(2): 89-95.

50. Klingensmith GJ, Pyle L, Nadeau KJ, et al. Pregnancy Outcomes in Youth With Type 2 Diabetes: The TODAY Study Experience. Diabetes Care 2016; 39(1): 122-9.

51. Grey $M$, Whittemore $R$, Tamborlane $W$. Depression in type 1 diabetes in children: natural history and correlates. J Psychosom Res 2002; 53(4): 907-11.

52. Lawrence JM, Standiford DA, Loots B, et al. Prevalence and correlates of depressed mood among youth with diabetes: the SEARCH for Diabetes in Youth study. Pediatrics 2006; 117(4): 1348-58.

53. Walders-Abramson N, Venditti EM, levers-Landis CE, et al. Relationships among stressful life events and physiological markers, treatment adherence, and psychosocial functioning among youth with type 2 diabetes. J Pediatr 2014; 165(3): 504-8.e1.

54. Levitt Katz LE, Swami S, Abraham M, et al. Neuropsychiatric disorders at the presentation of type 2 diabetes mellitus in children. Pediatr Diabetes 2005; 6(2): 84-9. 
55. Varni JW, Burwinkle TM, Jacobs JR, Gottschalk M, Kaufman F, Jones KL. The PedsQL in type 1 and type 2 diabetes: reliability and validity of the Pediatric Quality of Life Inventory Generic Core Scales and type 1 Diabetes Module. Diabetes Care 2003; 26(3): 631-7.

56. Nadeau K, Kolotkin RL, Boex R, et al. Health-related quality of life in adolescents with comorbidities related to obesity. J Adolesc Health 2011; 49(1): 90-2.

57. Mulvaney SA, Schlundt DG, Mudasiru E, et al. Parent perceptions of caring for adolescents with type 2 diabetes. Diabetes Care 2006; 29(5): 993-7.

58. Salamon KS, Brouwer AM, Fox MM, et al. Experiencing type 2 diabetes mellitus: qualitative analysis of adolescents' concept of illness, adjustment, and motivation to engage in self-care behaviors. Diabetes Educ 2012; 38(4): 543-51.

59. Brouwer AM, Salamon KS, Olson KA, et al. Adolescents and type 2 diabetes mellitus: a qualitative analysis of the experience of social support. Clin Pediatr (Phila) 2012; 51(12): 1130-9.

60. Copeland KC, Silverstein J, Moore KR, et al. Management of newly diagnosed type 2 Diabetes Mellitus (T2DM) in children and adolescents. Pediatrics 2013; 131(2): 364-82.

61. Wittmeier KD, Wicklow BA, Sellers EA, et al. Success with lifestyle monotherapy in youth with new-onset type 2 diabetes. Paediatrics \& Child Health (1205-7088) 2012; 17(3).

62. Shield JPH, Lynn R, Wan KC, Haines L, Barrett TG. Management and 1 year outcome for UK children with type 2 diabetes. Archives of Disease in Childhood 2009; 94(3): 206-9. 63. Nadeau KJ, Anderson BJ, Berg EG, et al. Youth-Onset Type 2 Diabetes Consensus Report: Current Status, Challenges, and Priorities. Diabetes Care 2016; 39(9): 1635-42.

64. Dujic T, Zhou K, Tavendale R, Palmer CNA, Pearson ER. Effect of Serotonin Transporter 5-HTTLPR Polymorphism on Gastrointestinal Intolerance to Metformin: A GoDARTS Study. Diabetes Care 2016; 39(11): 1896-901.

65. Dawed AY, Donnelly L, Tavendale R, et al. <em $>$ CYP 2 C $8</ e m>$ and $<$ em $>$ SLCO1B1</em $>$ Variants and Therapeutic Response to Thiazolidinediones in Patients With Type 2 Diabetes. Diabetes Care 2016; 39(11): 1902-8.

66. TODAY Study Group. A Clinical Trial to Maintain Glycemic Control in Youth with Type 2 Diabetes. New England Journal of Medicine 2012; 366(24): 2247-56.

67. Laffel L, Chang N, Grey M, et al. Metformin monotherapy in youth with recent onset type 2 diabetes: experience from the prerandomization run-in phase of the TODAY study. Pediatric Diabetes 2012; 13(5): 369-75.

68. Turner KM, Percival J, Dunger DB, Olbers T, Barrett T, Shield JP. Adolescents' views and experiences of treatments for Type 2 diabetes: a qualitative study. Diabetic medicine : $a$ journal of the British Diabetic Association 2015; 32(2): 250-6.

69. Christensen ML, Franklin BE, Momper JD, Reed MD. Pediatric drug development programs for type 2 diabetes: A review. J Clin Pharmacol 2015; 55(7): 731-8.

70. Nambam B, Silverstein J, Cheng $P$, et al. A cross-sectional view of the current state of treatment of youth with type 2 diabetes in the USA: enrollment data from the Pediatric Diabetes Consortium Type 2 Diabetes Registry. Pediatr Diabetes 2016.

71. Black J, White B, Viner R, Simmons R. Bariatric surgery for obese children and adolescents: a systematic review and meta-analysis. Obes Rev 2013; 14(8): 634-44.

72. Shah AS, D'Alessio D, Ford-Adams ME, Desai AP, Inge TH. Bariatric Surgery: A Potential Treatment for Type 2 Diabetes in Youth. Diabetes Care 2016; 39(6): 934-40.

73. Rubino F, Nathan DM, Eckel RH, et al. Metabolic Surgery in the Treatment Algorithm for Type 2 Diabetes: A Joint Statement by International Diabetes Organizations. Diabetes Care 2016; 39(6): 861-77. 
74. Inge TH, Courcoulas AP, Jenkins TM, et al. Weight Loss and Health Status 3 Years after Bariatric Surgery in Adolescents. N Engl J Med 2016; 374(2): 113-23.

75. Miras AD, le Roux CW. Mechanisms underlying weight loss after bariatric surgery. Nature Reviews Gastroenterology and Hepatology 2013; 10(10): 575-84.

76. Rubino F, Nathan DM, Eckel RH, et al. Metabolic Surgery in the Treatment Algorithm for Type 2 Diabetes: A Joint Statement by International Diabetes Organizations. Diabetes Care 2016; 39(6): 861-77.

77. Kaulfers A-MD, Bean JA, Inge TH, Dolan LM, Kalkwarf HJ. Bone Loss in Adolescents After Bariatric Surgery. Pediatrics 2011.

78. Danne T, Biester T, Kapitzke K, et al. Liraglutide in an Adolescent Population with Obesity: A Randomized, Double-Blind, Placebo-Controlled 5-Week Trial to Assess Safety, Tolerability, and Pharmacokinetics of Liraglutide in Adolescents Aged 12-17 Years. J Pediatr 2017; 181: 146-53 e3.

79. Klein DJ, Battelino T, Chatterjee DJ, et al. Liraglutide's safety, tolerability, pharmacokinetics, and pharmacodynamics in pediatric type 2 diabetes: a randomized, double-blind, placebo-controlled trial. Diabetes Technol Ther 2014; 16(10): 679-87.

80. Wong K, Potter A, Mulvaney S, Russell WE, Schlundt DG, Rothman RL. Pediatric Endocrinologists' Management of Children With Type 2 Diabetes. Diabetes Care 2010; 33(3): 512-4.

81. Pulgaron ER, Hernandez J, Dehaan $\mathrm{H}$, et al. Clinic attendance and health outcomes of youth with type 2 diabetes mellitus. Int J Adolesc Med Health 2015; 27(3): 271-4.

82. Brackney DE, Cutshall M. Prevention of type 2 diabetes among youth: a systematic review, implications for the school nurse. J Sch Nurs 2015; 31(1): 6-21.

83. Viner RM, Segal TY, Lichtarowicz-Krynska E, Hindmarsh PC. Prevalence of the insulin resistance syndrome in obesity. Arch Dis Child 2004; In Press.

84. Nguyen TT, Jayadeva V, Cizza G, et al. Challenging recruitment of youth with type 2 diabetes into clinical trials. J Adolesc Health 2014; 54(3): 247-54.

85. Jump Study. Type 2 Diabetes in Childhood: UK National Cohort Study (JUMP). 2017. http://www.rcpch.ac.uk/type-2-diabetes-childhood-uk-national-cohort-study-jump (accessed 28 January 2017. 